Last draft. Final version to appear in Journal of Agricultural and Environmental Ethics

\title{
TITLE
}

What Is a Recipe?

\author{
AUTHOR \\ Andrea Borghini \\ Department of Philosophy \\ College of the Holy Cross \\ 1 College Street \\ Worcester MA, 01610 \\ USA \\ Email: aborghin@holycross.edu \\ Phone: 508-793-3453
}

ABSTRACT. The ontology of recipes is by and large unexplored. In this paper, I offer a three-steps account. After introducing some key terminology, I distinguish four main options for a theory of recipes: realism, constructivism, existentialism, and the naïve approach. Hence, I first argue that recipes are social entities whose identity depends (also) on a process of identification, typically performed by means of a performative utterance on the part of a cook (e.g. "This is fettuccine Alfredo," "This is falafel"); thus, the best theoretical framework for a theory of recipes is a constructivist. Secondly, I argue that the identity of recipes can be grasped only by being suitably acquainted with the dishes that instantiate them, because of the impossibility to spell out recipes in details that would match a full-fledged dish; hence, the authority to establish the identity of a recipe rests on a process of apprenticeship. Finally, I argue that the identity of recipes and vicariously - of the dishes that instantiate them, rest on three factors: the expertise required on the part of the cook; authenticity (in turn based on the fit and approval rate of any purported rendering); and the open-ended character of recipes.

KEY WORDS: recipes; ontology; identity; authenticity in food. 


\section{What Is a Recipe?}

You learn to cook so that you don't have

to be a slave to recipes - Julia Child

Philosophers tackled important questions pertaining to the ethics of our dietary choices and the aesthetic worth of foods. However, many dimensions of eating remain by and large unexplored, such as hunger, the taxonomies of edible things, or metabolism. If we assess the status quo in terms of philosophical branches, the ontology of food is the least studied and remains nearly untamed. Recipes are an excellent topic to start covering the gap because of their their cultural, legal and economic relevance, their centrality to cuisine, and their intellectual sophistication.

Recipes are key tools in any culinary culture, instructing diners on how to prepare ingredients in a safe, nutritious, pleasing fashion. The escalating social recognition of recipes has recently found expression also in the Unesco Intangible Cultural Heritage list. The first culinary items to make the list, in 2010, were the Mediterranean diet (as adopted in Cyprus, Croatia, Italy, Spain, Greece, Morocco, and Portugal), the gastronomic meal of the French and traditional Mexican cuisine, followed by Washoku (traditional dietary cultures of Japan) in 2013; additional items, such as the traditional art of Naples "pizzaiuoli", are under consideration. Each of these items rest on recipes for its identity, as the notion of a cuisine or culture's gastronomy encompasses a set of related representations, of which recipes are key elements (for some classic study, cfr. Fischler 1988 and 1980).

Not only recipes now benefit from the legal and socio-economic advantages of the items in the Intangible Cultural Heritage list; recipes associated with so-called geographical indications, e.g. gorgonzola and Parmigiano cheese, have been protected by intellectual property right for a long time (cfr. Borghini 2014a). Geographical indications comprise a key sector of the food trade to date, with over ten thousands geographical indications being marketed in 2009 for an estimated value of over US\$50 billions (see Yeung 2014).

The increasing importance of recipes is also showcased and fostered through the media. Cookbooks have come to be a major sector of the publishing industry. A simple search on the book section of Amazon.com (as of April 19, 2014) returns 106,137 entries for cookbooks; just above twenty percent of the entries for philosophy books, which returns 521,809 . The data are even more staggering if we consider that only a handful of the 106,137 entries were written before the thirteenth hundreds. The oral transmission of recipes, by means for instance of rhyming descriptions (Limberg 2009), was arguably pivotal to the thriving of the human species. In the past two centuries, then, the affirmation of restaurants and restaurant menus, has importantly contributed to the ascent of recipes and, probably, also to the custom of writing them down. Through recipes we can also reconstruct social history (cfr. Shapiro 2008). Recipes TV shows and media are now rendering recipes more and more akin to items of popular culture like songs and movies.

Finally, food issues are not only increasing in popularity; in a very urgent sense, they are also at the forefront of important global health and environmental problems. A 
theory of recipes helps policy makers, thinkers and educators to be better conversant in these problems. For instance, dieting is best characterized in terms of ingredients and recipes, so that addressing obesity as well as eating disorders requires a better understanding of recipes. Or, to offer another example, the biodiversity found within foods consumed by humans was preserved and fostered also thanks to recipes; a theory of recipes would help us reconstruct an important chapter of human evolution and also to evaluate the biodiversity within current human diets.

The clarification of the theoretical issues surrounding a theory of recipes therefore has enormous practical importance. As we shall see, it raises some difficult philosophical questions too. Yet, the importance of recipes in the public sphere is not matched by an adequate scholarly examination. With the exception of some cursory remarks (Telfer 1996, especially ch. 3; Korsemeyer 1999; Borghini 2014b and 2011), no discussion of recipes has been put forth in the philosophical literature. More in general, to date no book or scholarly article offers a theoretical account of recipes. On a par with shadows, holes, smells, or gestures, recipes are part and parcel of that ontological scaffolding withouth which it would be difficult to imagine our lives; but, in a grand scheme of the world such as that which is taught in schools, recipes appear as a lesser kind (Casati \& Varzi 2007), leaving the stage to kinds of things such as electrons, water, cells, animals, stars, people, money, and States. In this paper I start covering the theoretical gap by offering a threesteps account of recipes.

The paper begins by introducing some key terminology $(\S 1)$ and distinsguishing four possible theoretical frameworks for recipes: realist, constructivist, existentialist, naïve $(\S 2)$. Next, I argue that recipes are social entities whose identity depends (also) on a process of identification, typically performed by means of performative utterance on the part of a cook (e.g. "This is fettuccine Alfredo," "This is falafel"); thus, the best theoretical framework for a theory of recipes is a constructivist. (§3) Secondly, I argue that the identity of recipes can be grasped only by being suitably acquainted with the dishes that instantiate them, because of the impossibility to spell out recipes in details that would match a full-fledged dish; hence, the authority to establish the identity of a recipe rests on a process of apprenticeship. (§4). Finally, I argue that the identity of recipes and - vicariously - of the dishes that instantiate them, rest on three factors: the expertise required on the part of the cook; authenticity (in turn based on the fit and approval rate of any purported rendering); and the open-ended character of recipes ( 55$)$.

The account I defend here has important consequences also for the ongoing legal debates about the identities of specific recipes, including the best framework within which to assess the identity of geographical indications and the current dispute within the Translatlatic Trade and Investment Partnership concerning the identity of specialty foods As I shall illustrate in $\S 3$, these debates aim to legislate about recipes by resorting to forms of realism about recipes, thereby obliterating the processes of negotation that - I argue - characterize the identity of recipes. As such, the debates rest on a fundamental misunderstanding of the nature of recipes that risks of providing unfair advantage and escalating social tensions.

\section{§1 DISHES AND RECIPES}


"Recipe" and "dish" are often used interchangeably. The two words, however, may stand for two kinds of entities. For this reason, I will bind "recipe" to one kind of entity and "dish" to another. In a nutschell, a dish is the stuff, a recipe is the idea. More precisely, a dish is a specific concotion of (typically perishable) edible stuff, such as those specific actions that led to this slice of pizza sitting on my kitchen counter. On the other hand, a recipe - in first approximation - comprises the array of repeatable aspects of a dish whose replication would deliver a dish of the same sort.

Are there things that we eat that are not dishes? I tend to have a permissive understanding of dishes. According to it, anything that is ready for someone to be eaten up is a dish. ${ }^{1}$ Not only what we find in a restaurant menu or what we cook at home after an elaborate preparation is a dish. Some food products are dishes (e.g. chocolates, candies, cheeses, cured meats, pickled vegetables, cured olives, fresh pasta); foodforaging is a manner of procuring dishes; cherries picked and eaten from a tree are a dish; having a drink (e.g. coffee, tea, cocktail) is having a dish. I take this view because it avoids one major issue: distinsguishing between what is ready to be eaten up and is not a dish and what is ready to be eaten up and is a dish. I see no reasonable principled manner of drawing such a distinction and I see no drawbacks for having a permissive understanding of dishes.

It is at this stage important to dispel also a source of potential confusion in the discussion of dishes and recipes. When speaking of a dish or a recipe, at times we refer merely to the item that is consumed - say, a slice of pizza; in other contexts, however, we refer to that item plus the relevant events that brought to its realization; the relevant events, in turn, may be limited to the action of the cook (say, all the toil and labour that went into preparing the slice) or, rather, the events may comprise a more extended series (e.g., the farms where the grains, the tomatoes, and the milk were produced). I call the latter the extended concept of a recipe or dish. It is the extended version of a recipe that I discuss in this paper. It shall be clear that I am not arguing for the ontological priority of the extended concept over the non-extended one. More modestly and aptly, the extended concept, being richer, allows for a more nuanced discussion of the subject. ${ }^{2}$

Not every dish need exemplify some recipe. In principle, the procedure that delivers any dish has some aspect to it that could be replicated. However, some dishes and the procedures that deliver them - remain neglected. It may be because they are

\footnotetext{
${ }^{1}$ Note that I am not claiming that anything that is edible is a dish. The characterization offered here is relational, that is a dish is a dish-for-someone-in-a-context. At any moment, there is usually some edible stuff in the vicinity of an agent that the agent would never see as "ready to be eaten up" (e.g. clay, worms, pine shoots).

${ }^{2}$ The extended concept of a recipe may suggest to some readers that a theory of recipes as kinds of actions - paralleling Davies's theory of musical works as actions (2004) - is most apt. In the rest of the paper, however, I provide an account that diverges from Davies's, in two main respects: first because in my view the identity of recipes rests on a performative utterance, rather than on the mere actions that bring to the making of a dish; secondly, and more importantly, because the identity of recipes depends on collective judgments of authenticity, not simply on the actions that bring to the making of a dish.
} 
unappealing; but, more circumstantial reasons could intervene too, for instance the conditions in which the dish is prepared may be truly exceptional (e.g. a dish prepared while stranded on a highway or during an expedition to the Antartic) and replicating it is not worth it. Thus, only selected dishes enter the ranks of recipes. How the selection is operated and what features are deemed as salient to be included in the recipe is part and parcel of the very ontological conundrum of recipes, which I discuss in the next section.

A recipe may exist before having been instantiated into a dish or, in extreme cases, without ever being instantiated. In some fortunate cases, even before executing a novel recipe for the first time, a cook had the recipe. Other times, the dish and the recipe come to be simultaneously - as the chef makes a dish, she is also (perhaps even accidentally) discovering the recipe. Other times still, the recipe is derived after a few trials and errors. So, there is no one-one correlation between dishes and recipes, and the existence of dishes can precede, be simultaneous, or follow the existence of the respective recipes.

The relationship between dishes and recipes poses a problem that lies at the core of any analysis of recipes. The problem concerns the partition of dishes into recipes. Suppose you have mapped out all the dishes that there are; how many recipes do those dishes instantiate? Call this the hard problem of recipes.

To address the problem, it is useful to introduce some additional terminology, borrowed from contemporary metaphysics, and say that the relationship between dishes and recipes is the one of instantiation or exemplification; that is, a dish can be an instance of a recipe or, alternatively, an example of a recipe. This slice of pizza - a dish - is an instance of pizza's recipe; last week another slice of pizza was prepared, which has now been all eaten up.

In the next section $(\S 2)$, we survery four theoretical approaches to recipes and contend that only two of them - realism and constructivism - are eligible to tackle the hard problem of recipes. Upon showing some shortcomings of realism, a constructivist framework will be then provided in the remaining of the paper.

\section{\$2 FOUR THEORIES OF RECIPES}

Borrowing the results of long-standing research in the social sciences (especially anthropology and history), four main options for a theory of recipes turn up: realism, constructivism, existentialism, and the naïve approach. Let us briefly review each of them. ${ }^{3}$

${ }^{3}$ Before moving on, some words about beverages and foods involving fermentation may be useful. I see no principled reason to exclude items such as wines, spirits, vinegars, pickles, dried meats, and cheeses from the list of recipes. Wines, for instance, can be conceived as recipes that are executed once a year by expert makers, following specific scripts. The literature on the philosophy of wine is, by now, quite extensive and includes a few discussions of the ontological status of wine too. For some recent contributions, cfr. Burnham and Skilleas 2012, Borghini 2012, Scruton 2009, Smith 2007, and Todd 2010. Some contributions to the philosophy of beer (Hales \& Jackson 2008) and whisky 
(i) Realism. Realism is the most ambitious position. According to the realist about recipes, the identity of a recipe depends solely on the ingredients and the procedure for preparation. We can distinguish two strains of realism. The first is hard-core realism, which draws from the classic work of Boorstin (1964) and sees a recipe as a practice that is in its essence unchanging; a recipe, under this strain, is essentially defined by a list of ingredients and procedures that recur in any occasion. An example bringing evidence favorable to hard-core realism is pizza Margherita, which arguably essentially involves tomato, mozzarella, olive oil, salt, basil, and pizza dough; nothing can be pizza Margherita unless it does not involve those ingredients.

Hard-core realism faces important challenges when trying to accommodate a large variety of recipes, whose evolution involves dramatic changes in ingredients and procedures, so that possibly none of the initial ingredients and procedures are preserved throughout the evolutionary process. Consider, for instance, cheweing gum, a recipe whose ingredients have changed across time. Today, the gum base is no longer chicle (or tar or mastic), but typically synthetic rubber; and there are several options for the sweeteners (e.g. sugar has been substituted by corn syrup or beet juice), softeners (e.g. glycerin or vegetable oil), and flavorings. Hence, it is unclear how hardcore realism can account for the intuition that chewing gum is the same recipe, despite its changes throughout time. In other cases, procedures change, because of the introduction of new technologies; for instance, consider the differences between a handmade whipped cream and a whipped cream made by using a mixer. Hard-core realism seems to require a notion of identity for recipes that is too strict and thus unable to accommodate the ways in which recipes are de facto identified.

The second strain of realism makes room for the possibility of change in the defining elements of a recipe and we shall label it mild realism. According to mild realism, even though all the ingredients for chewing gum changed over time and may change based on the producer, it is still the case that, for each version of the recipe, there is a core of ingredients and procedures that fixes the identity of the recipe.

Mild realism is arguably endorsed in devising the identity conditions of foods protected by a geographical indication, such as Parmigiano and Rocquefort, as well as in the case of foods enjoying particular attention, such as the official recipe for pesto alla genovese adopted for the Genoa Pesto World Championship (cfr. Borghini 2014a). Despite its plausibility, mild realism falls short of capturing the identity conditions of a good number of important recipes. For instance, some recipes - e.g. a chef's salad or a house pizza - owe their identity to the sort of person preparing them rather than to the ingredients or the procedures. In general, the idea that a recipe depends on the fiat of the cook is entirely in keeping with practice, but mild realism is unable to accommodate any case of this sort.

(ii) Constructivism. The constructivist maintains that recipes are the outcome of a selection process ultimately guided by human fiat (cfr. Sims 2009, Germann Molz 2004, and Jackson 1999). ${ }^{4}$ The identity of a recipe, that is, does not rest on specific ingredients

(Allhoff \& Adams 2010) have been produced as well, while cheese - qua cultural item remains so far mostly studied by anthropologists and historians.

${ }^{4}$ Some parallel disputes between realists and constructivists in the philosophy of biology are of importance to frame the debate on recipes. Although space limitations do not allow 
or procedures; what matters, rather, is that whoever produces the recipe recognizes it as such. ${ }^{5}$ In this paper, I shall elaborate a full-fledged constructivist account of recipes. In the next section, I argue that the identity of recipes is tied to a process of identification, typically performed by means of a speech act, more precisely a performative utterance, on the part of a cook - e.g. "this is Fettuccine Alfredo." Next, I contend that the identity of recipes can be grasped only by being suitably acquainted with the dishes that instantiate them, because of the impossibility to spell out recipes in details that would match a full-fledged dish; hence, the authority to establish the identity of a recipe rests on a process of apprenticeship. In the final section, I will round my account by arguing that the identity conditions of a recipe rest on three core factors: expertise, authenticity, and open-endedness. A recipe is the product of an agent with suitable expertise who declares to be imitating a rendering of the recipe.

(iii) "Existentialim." Bits of the existentialist philosophy have been adopted in the contemporary debate on tourism and authenticity (cfr. Wang 1999 and Sims 2009: 324-325), and they can be employed to suggest a sui generis understanding of recipes. Because gastronomic experiences contribute to a sense of self, recipes are part and parcel of a quest for the realization of an authentic self. On a par with the constructivist, the existentialist maintains that the identity of recipes depends on a human fiat; but, the existentialist limits the construction to a dialogue that is relevant to an individual person, while a constructivist would typically underline the social dimension of recipes.

While the existentialist approach to recipes is useful to the study of phenomena such as gastro-turism and to analyze categories such as authenticity in gastronomy, it is unfit for providing a theoretical framework of use to policy makers and lawyers, and to address normatively issues concerning global health, or cultural and environmental preservation. The existentialist approach, indeed, makes it impossible to develop an account where recipes are social entities. While it is important to frame and study subjective responses to cultural items such as recipes, the present aim is to deliver a theory where recipes are cultural construct that are created and managed by collectivities. After all, the inventor of a great majority of recipes - beer, wine, bread, pasta, mozzarella, ketchup, ... - is unknown and the recipes do not belong to anyone, but are rather tended by large collectivities. For those reasons, I shall instead leave the existentialist option on a side.

(iv) Naïve Approach. Naïve approaches to authenticity have been proposed to explain consumer's culture (Cohen 2002 and Coombe 2009). In this perspective, recipes are products of popular culture, emerging out of often inconsistent judgments; there can be no genuine arguing about the identity of a recipe that goes beyond taking stock of consumer's opinions. The naïve approach is suitable to provide a descriptive report of how recipes are sorted out and to derive important speculative conclusions about the

to bring in full-fledged comparisons, the reader may want to consult at least also the debate on the ontological status of human races and the debate on the ontological status of sexual categories as applied to humans.

${ }^{5}$ Sims (2009: 234), drawing on (Jackson 1999), comes close to suggest this view in her discussion of authenticity: "instead of talking about «authenticity», we should focus upon «authentification», which is the process whereby people make claims for authenticity and the interests that those claims serve." (Sims 2009: 324) 
reports. But, like the existentialist approach, the naïve approach is unsuitable for delivering a theoretical proposal that has sufficient normative force to sustain legal and cultural disputes about the identity of recipes. Such a normative requirement is needed if the account of recipes can be used to support or discredit legal claims about the identity of products, such as those that ground decisions about geographical indications and the Unesco Intangible Cultural Heritage list.

\section{§3 DISHES AND SPEECH ACTS}

According to a realist, whether a dish instantiates a recipe depends exclusively on the features of the dish and not at all on a human fiat. Thus, if a class of dishes instantiates the same recipe, this must be because the dishes share some common elements. Looking more in depth into the realist position, three models for a realist relationship between dishes and recipes suggest themselves: one horizontal, one vertical, and a hybrid one. According to the horizontal model, which is broadly inspired by the Aristotelian tradition, all dishes instantiating the same recipe share at least a property (and, typically, a few properties), that is an ingredient or a procedure. For example, all instances of pizza share flour and yeast. According to the vertical model, which draws on taxonomic lessons suggested by evolutionary theory, dishes instantiating the same recipe share a common origin, but need not share any common horizontal property. For example, all instances of pizza originate out of a common ancestor dish, although we may find pizzas of all kinds in such a way that no set of common horizontal properties can be devised. Finally, according to a hybrid and milder model, dishes instantiating a recipe need not universally share common horizontal properties, though they comprise families of common horizontal properties or of common origin, in such a way that each dish falling under a recipe is linked to one or more dishes that also fall under that recipe.

None of the models seem to adequately restitute the ontology of recipes. The first model is falsified by most of our recipes; for example, pizzas have been made with and without all sorts of ingredients, including flour and yeast. ${ }^{6}$ The second model poses too weak of a constraint over the identity of a recipe; for example, the instances of chicken and veal parmigiana have the same origin of the instances of eggplant parmigiana, but

\footnotetext{
${ }^{6}$ It may be rebutted that pizza is not a recipe, but a cluster of recipes. The rebuttal would fall short of its target, though, as many other examples can be produced: ice cream (including specific versions, e.g. strawberry ice cream), croissant, lemon cake, ... Nonetheless, the rebuttal points the finger at an important question, related to the hard problem of recipes: how to tell a single recipe from a cluster of recipes? I see no principled manner to address the question. But, I can point out that similar puzzles exist for musical and literary works. Is the Happy Birthday song the same song also when recited in languages other than English, or does each language have its own "Happy Birthday" song? Is the Exodus the same book in all languages, or does each language have its own Exodus book? Copyright laws tend to favor the first option, which is ontologically more parsimonious. But, that is not the only take on the matter; think for example of the Quran, which many would argue cannot exist in another language form, or think of some notable poems such as Beowulf, the Bhagavad Gita, or the Commedia.
} 
arguably all the instances do not comprise one same recipe. The third model seems the most plausible and I do endorse a constructivist take on it. In its realist version, indeed, it retains a major problem of realism about recipes. Whether an array of dishes instantiates one and the same recipe is not simply a matter of sharing certain horizontal or vertical features. Recipes often denote social boundaries that do not rest on any plausible story about corresponding natural discontinuities. For example, the difference between ice cream and gelato can hardly be explained in terms of a well-formed criterion based on ingredients or preparation. Or, the differences between chickpea-flour-based cakes known as cecina, torta, socca, farinata, fainè, faina can hardly be accounted for on the basis of differences in the recipes. Some social boundaries - that is - exist precisely in order to have some boundaries. And recipes can aptly be used to draw such social boundaries. Furthermore, as we have seen in $\S 2$, the three models cannot accommodate cases of recipes whose identity deliberately depends on the fiat of some cook, such as a chef's salad or a house pizza.

The shortcomings of the three models can be surpassed by postulating that recipes depend on a process of identification, typically performed by means of a speech act. More precisely, we can help ourselves here by invoking the importance of performative utterances - in the words of J.L. Austin - in the making of recipes. Performatives are typical of social entities, such as marriages or currency. By uttering: "I do," a speaker makes a marriage happen; by stating "This note is legal tender for all debts, public and private," the United States Federal Reserve makes a piece of currency out of a piece of paper. Recipes, that is, must contain a performative utterance; e.g. pointing at a dish, a script, a painting, a video, or opening up a description, the cook would declare: "This is the recipe for strawberry gelato," "This is the recipe for cecina;" in cookbooks we are accustomed to read such an utterance as a disguised title for the recipe, e.g. "Recipe for strawberry gelato," "Strawberry gelato for four," or "Strawberry gelato."

It is important to qualify my suggestion, in order to avoid a potential confusion? Alike marriages and currencies, recipes depend upon a performative utterance for their existence; but the identity of recipes and of the dishes that instantiate them depends also on factors that are foreign to weddings or currencies. Thus, I am not proposing that recipes are ontologically on a par with contemporary currencies or marriages, which seem to depend entirely on human fiat; rather, I am arguing that recipes depend constitutively, but not entirely on some human fiat. In as much as a recipe is an artifact, which bears a label and is apt of being reidentified at different times and places, a recipe cannot exist unless someone declares its existence; this is why they are constitutively dependent upon some speech act. On the other hand, as we shall see, recipes require also apprenticeship ( $\$ 4)$ and their identity rests on a complex process of negotiation ( $\$ 5)$. That is, the relationship between the sort of agent uttering the performative and the utterance is quite specific in the case of recipes: the agent must be qualified to cover the role required to succesfully label a recipe, and such qualification is acquired through a process of apprenticeship.

${ }^{7}$ I thank an anonimous referee for prompting this qualification. 
The constructivist approach will concern not only recipes, but also dishes. The author(s) of a dish must declare the intention to be replicating a certain recipe (e.g. "This is an instance of gelato," "This is an instance of cecina"). Whether the dish is or is not a rendition of a recipe will depend also on such declaration of intention; but, it does not depend solely on the declaration. As we shall see (§5.2), the performative selects the relevant class of comparison for the dish as well as indicates the appropriate judging body. By linking dishes to recipes, cooks and diners contribute to the evolution of the recipe. In a similar vein, whether a certain musical performance is or is not the cover of another musical performance depends, at least in part, on the declaration of intent of the players.

Solving - or dissolving - the hard problem regarding the relationship between dishes and recipes is one of the main tasks of a theory of recipes. Realism is looked at with suspicion by most contemporary scholars working on food. This is because recipes are only rarely available in coockie-cutter guises ${ }^{8}$ and, as we shall see, they are arrived through complex processes of negotiation. There is one important sphere, though, where realism seems to have a grip, and that is with so-called geographical indications (GIs). GIs protect the essential contribution of a geographical location in the making of a product. Such a contribution is provided by special climatic, geological, and biotic conditions along with a specific know-how (cfr. Borghini 2014a). GIs were introduced to protect goods of all sorts, including jewellery, textiles, and handicrafts. Most GIs, however, are food products. Early specimens include wines such as Chianti Classico (specifically designated since 1716), Tokaj-Hegyalja wines (1730), Champagne (invented at least in 1531, defined in 1662, and protected from 1891), and Port (1756). GIs are today regulated under Articles 22 and 23 of the TRIPs agreement (the agreement on Trade-Related Aspects of Intellectual Property Rights), signed in 1994 within the WTO by 117 countries. According to Article 22.1:

Geographical indications are, for the purposes of this Agreement, indications which identify a good as originating in the territory of a Member, or a region or locality in that territory, where a given quality, reputation or other characteristic of the good is essentially attributable to its geographical origin.

GIs, which are treated as intellectual property rights, are identified through some essential properties of the protected items. To offer an example, consider Chianti Classico wines. A wine can be considered Chianti Classico only if the grapes from which it originated were grown in specific portions of nine townships in Central Tuscany, between the provinces of Florence and Siena in order to qualify as Chianti Classico, a product must fulfill a relatively long list of requirements, including grape variety, fermentation techniques, organoleptic aspects, and bottling restrictions. To a realist, those requirements define the recipe for Chianti Classico wines and indicate properties of the wine that are objectively verifiable.

If what I have argued in this section is correct, realism about recipes is ill-founded

\footnotetext{
${ }^{8}$ Notable exceptions are so-called "signature dishes," such as Bottura's Chicken chiken where are you?
} 
and realists about GIs fall short of being able to defend their position. Constructivism, on the other hand, points at the fact that guidelines for producing Chianti Classico wines have changed considerably over the years and are arrived at through a process of negotiation that is contentious, tedious, and made of compromises. While for practical purposes it is useful to have a list of properties that fix the identity of the recipe, in a realist fashion, such a list is arrived at through a deliberate decision among several options; that is, the list depends by and large on the fiat of those invested in making the list.

\section{\$4 RECIPES AND APPRENTICESHIP}

In recent years an impressive amount of cookbooks, filled with recipes, was published. The shift indicates a rising social relevance of food. But, implicitly, it suggests a twofold difficulty in disentangling recipes. First of all, we have no standard notation for recipes, so that different renditions of a same recipe will specify different details. Secondly, even if we would have a standard notation, it would remain impossible to record all aspects of a dish on paper in such a way that the recipe contains all the properties of a dish. On the contrary, recipes are doomed to be elliptic descriptions of dishes, and it is precisely in virtue of this that recipes can adapt to circumstances.

To dig deeper into this issue it is instructive to draw on the distinction between allographic and autographic forms of art introduced by Nelson Goodman (1976). ${ }^{9}$ Allographic works can be represented by a language other than the one in which they are expressed. For instance, Dante's Commedia is an allographic work because we have a linguistic notation that can represent the (most salient aspects of the) work, which in itself would be a speech performance. Bach's Golberg Variations are another plausible case in point - assuming that the score represents their most salient features. On the other hand, an autographic work cannot be expressed other than by itself. Leonardo's Monnalisa is autographic; although it can be forged or reproduced in a picture or a video, the original copy is the work; everything else is at best imitation. So, the question arises of whether cooking is allographic or autographic.

Recording of recipes is by now quite standardized. Similarity in types of equipment across kitchens of the globe is increasing, so is the availability of translation schemes for units of measure; the format of a recipe, furthermore, is also becoming increasingly standard - a list of (incresingly standardized) equipment and (incresingly standardized) ingredients, with quantities and serving, plus details of procedures. For many recipes - not only industrial products, but also foraged foods, dishes that require simple cooking procedures, or pastries - these standards are good enough to guarantee the delivery of a dish that replicates the recipe. Thus, some recipes are allographic.

The adoption of a standard, however, is possible only for some recipes and under rigorous circumstantial assumptions. To offer an essay of how difficult is to keep high standards for reporting recipes, consider the identity of ingredients and the taxonomies of

\footnotetext{
${ }^{9}$ I shall stay away here from the question of whether cooking can be an artform. For some chief contributions, cfr. (Telfer 1996) and (Korsemeyer 1999).
} 
foods upon which the identity of ingredients rests. First of all, the very concept of an ingredient seems to escape a clearcut understanding. In a restaurant menu, spices, salt or sugar are not typically listed (at least in their entirety) among the ingredients. In labelled foods, the components of a recipe below a certain percentage of the total product - such as yeasts and bacteria - are often not listed or fully specified; thus, for instance, even in European markets - where the doctrine of substantial equivalence (cfr. Borghini 2014c) has not been endorsed - items containing GM yeasts typically do no report the presence of a GM ingredient. The issue is not only a practical one. How small shall an ingredient be? Shall nanoparticles be regarded as ingredients, as some have argued (cfr. Gatti \& Montanari 2007)? Is the ingredient relation transitive - for instance, shall bacteria or yeasts found within ingredients be regarded as ingredients? These questions suggest that the most basic units of a recipe are uncertain in a way that the most basic units of music aren't. Secondly, the classification of foods is a messy business and it affects how we identify ingredients. Consider for instance bacteria. The specimens found on a rind of Parmigiano arguably contribute to the taste of the cheese; thus, the identity of Parmigiano depends upon its bacteria. However, the classification of bacteria is far from uncontroversial, which leaves us baffled when trying to identify Parmigiano (Ereshefsky 2010, Franklin 2007, and Doolittle \& Papke 2006). Similar arguments apply to virtually all foods that contain bacteria and are a threat to the preservation of the integrity of traditional foods as well as to the transparency of food labels. Or, think of the way in which varieties of plants are identified. Even easy cases such as specific varieties of cloned plants (e.g potatoes and apples), may have considerably different gustatory properties depending on the method of production, the area of production, or the seasonal climate; at a market, the criteria of identity for potatoes and apples do not take those gustatory differences into account; but, shouldn't they? Cooks and diners do care greatly about them, after all. Thirdly and most importantly: which aspects of a dish are necessary to the identity of its recipe and which are accidental? While to fill in many details of a dish for the purpose of generating a memory may be very useful, to fill in all the details would pose questionable restrictions over the cook's creative process. A demanding list specifying - say - the brand of cooking and eating tools would render most modifications illegitimate (if one regards a recipe as an entity whose identity is fixed once and for all); or, conversely, too sparse a list would suffice to make most modifications legitimate. It seems the responsibility and enjoyment of the cook to find what's most appropriate to her circumstances. What a recipe is cannot be pointed out by providing a cluster of necessary and sufficient conditions specifying exactly which ingredients, actions, or settings are proper (for a parallel with musical works, cfr. Gaut 2000 and Kivy 1995).

The lack of a standard notation and - most importantly - the elliptic nature of recipes are bad news for what we shall call a Platonist conception of recipes, borrowing a terminology that is customary in the ontology of musical works (though only vaguely reminiscent of the philosophy of Plato). The Platonist holds that entities essentially occupying a spatio-temporal region (such as this circular table) depend for their existence upon entities that can be conceived independently of a specific spatio-temporal region (such as the idea of a circle). For instance, in the philosophy of music we find a well- 
established Platonist ontology, according to which musical works are ethernal entities, ideally captured in their score (cfr. Kivy 1993 and Dodd 2007); musical performances, in this perspective, exist vicariously to scores. A Platonist ontology of recipes contends that dishes are but imperfect representations of recipes.

The Platonist conception of recipes faces an important challenge, which arises from the relationship between recipes and dishes. In fact, unlike movies, literary works, and certain musical works, it seems that recipes are not autonomous entities, but are vicarious of dishes. As I remarked above, it is impossible to spell out recipes in details that would match a full-fledged dish. If so, then it is allegedly impossible to gain full acquaintance with a recipe, unless we have experienced a dish instantiating that recipe. To clarify, I am not claiming that the recording of a recipe cannot precede in time the making of any dish instantiating that recipe; there are famous cases of cookbooks filled with "recipes" that have never been prepared, and cooks occasionally claim to come up with a "recipe" before ever preparing it. Nonetheless, I am contending that until a recipe has not been realized, we can only speak of it as an entity that could exist in potency, but that is not yet existing. Recipes, after all, characteristically emerge out of a trial-and-error process, which involves the preparation of several dishes. A recipe that was never tried out is a recipe-in-potency, an entity very close to being a recipe, but not quite a recipe yet.

I hence side with the so-called Aristotelians when it comes to recipes: dishes, that is entities occupying specific spatio-temporal regions, are as fundamental as recipes, that is entities occupying no specific spatio-temporal region. A recipe is existentially linked to some dish instantiating it: no such dish, no recipe. Knowledge of the recipe hence requires acquaintance with some dish instiatiating it and, typically, a process of apprenticeship that would fine-tune our acquaintance. On the importance of the process of apprenticeship we shall come back in the next section.

Since a recipe cannot fully substitute for any dish, through a recipe we have a script suggesting how to imitate a dish, not how to duplicate it. In some cases, e.g. foods produced in accordance to a standardized formula such as Hydrox cookies, we come close to a relation of duplication between different instances of a same recipe; however, those are limit cases, unsuitable to represent the great majority of the recipes we have. Hence, the (ideal) relationship between different instances of a same recipe is not strict identity, typically spelled out in terms of Leibniz's Law via the two principles of indiscernibility of identicals (if two entities are identical, then they have exactly the same properties) and identity of indiscernibles (if two entities have exactly the same properties, then they are identical); rather, different dishes instantiate a same recipe when they stand in appropriate relations of imitation, to be further discussed in the next section. Analogously, in music we speak of interpreting, covering, rendering a certain musical work (cfr. Varzi 2013).

At best, then, cuisine seems to occupy an intermediary position between allographic and autographic forms of art. An analogy may be drawn here with architecture, where a standard terminology cannot fully substitute the experience of the building (on the status of architecture see also Goodman 1976: 221). Several authors, 
indeed, have stressed the irreducibility of the culinary experience, claiming that food cannot adequately be represented by anything other than itself (cfr. also Telfer 1996, ch. 3 ). If that is the case, then cuisine is an autographic form of art (cfr. Perullo 2012).

\section{§5 EXPERTISE, AUTHENTICITY, AND OPEN-ENDEDNESS}

Recipes depend upon some performative utterances and are entrenched with the dishes instantiating them in such as way that, as the dishes keep coming in, recipes evolve. But, when is it that a dish imitates another dish (or class of dishes) in such a way that they instantiate the same recipe? I deal with this question in three parts. First, I address the expertise required in order to be able to imitate a dish. Secondly, I examine the authenticity of recipes. Thirdly, I consider the ontological status of recipes vis-à-vis their open-endedness.

\section{\$5.1 Expertise}

Recipes are receptacles of collective memories, trasmitting information about how to prepare and - in some cases - how to consume a dish. Now, a typical recipe will contain a list of ingredients and a set of ordered procedures that must be followed in order to deliver a dish that instantiates the recipe. But, as I argued above, this information is elliptic for additional details that the cook must be able to fill in by herself. Call those details the implicit residue of a recipe. Part of the implicit residue is information that could be conveyed descriptively, but that is deemed superfluous; another part, instead, rests on non-verbal abilities of the cook, which are acquired through apprenticeship. It is a key aspect of a recipe that the memory it carries forward must be transmitted not only through a book or a story, but also through an appropriate apprenticeship. In order to deliver a dish that instantiates a given recipe, then, a cook must possess expertise in the relevant abilities required to prepare the dish. Again, we can draw here a parallel with musical works. No matter how much detailed, the notation for a musical work is not the performance; in order to learn how to play a piece, an apprenticeship comes in handy. But, again, while in music a few key variables (such as notation and the instruments) have been subject to some sort of standards, variety abounds in the kitchen.

Cooking requires a wealth of skills, some of which do transfer to other recipes as well, while others are more specific. Recent literature on expertise suggests that there are different dimensions to acquired skills and abilities. In particular, Collins (2013) distinguishes four dimensions: exposure, development, natural talent, and esotericity. (i) Exposure. In order to gain expertise of a certain domain, an agent must be exposed to repeated experiences within the domain. In the case of recipes, exposure requires apprenticeship. In order to make pizza, a cook should have apprenticed with someone who knew how to make pizza, or have performed sufficiently similar tasks (e.g. breadmaking, focaccia-making). ${ }^{10}$ (ii) Development. Becoming a skillful cook (or, more

\footnotetext{
${ }^{10}$ Today's media offer the opportunity to apprentice also by watching a video on the Internet or on TV. It is, however, important not to overestimate the capacity of contemporary food shows to transfer skills to their viewers. There seems to be a stark
} 
simply, skillful at concocting some specific dishes) is a developmental process, which is often divided into separate stages, like the different belt colours wore by students of karate. Delivering a certain recipe (e.g. a well-made slice of pizza), hence, can be a matter of degree and a matter of time. (iii) Natural talent. Rolling a pizza dough, slicing potatoes, dressing salads, churning ice cream ... Plenty of skills are needed to cook. Some we may be equipped to do, while for others we may lack the necessary strength or bodily endowment. Moreover, some people demonstrate more talent than others in accomplishing certain tasks, even if they have seldom or never trained in them. Clearly, how a recipe will be imitated will depend on which natural talents are deployed by the cook. (iv) Lastly, skill transfer has an esoteric aspect to it too. When an expert pizza maker shows an apprentice how to roll the dough, not every single movement can be mentioned or explained. How good at imitating a master one is depends also on the extent within which one can capture what is left implicit in the agency of the master.

Finally, table companions, too, may have to learn specific skills in order to enjoy a recipe. For example, eating ramen requires ability in handling chopsticks; eating spaghetti requires ability in handling a fork (and, to some, a spon too); eating injera requires ability in eating with hands; etc. In fact, in restaurants that aim at serving authentic foods, servers or cooks tell clients how to enjoy the food.

\section{\$5.2 Authenticity}

Literature in the philosophy of food, starting at least with Telfer (1996), stressed that food is transient and that taste is a destructive sense, that is it needs to destroy its object of perception. Additionally, as I argued above, it is futile to apply Leibniz's Law to dishes because our criteria of identity for dishes overlook many differences between ingredients and methods of preparation. Whether two dishes instantiate the same recipe is not a matter of strict identity, but of imitation. Relationships of appropriate imitation are typically framed in terms of authenticity: a dish is said to be or not to be an authentic rendition of a recipe when it stands in an appropriate relationship of imitation with other dishes that instantiate the recipe. Now, imagine that you want to make pizza this evening - your first time making pizza on your own. You have the written recipe from a reliable source and you have apprenticed before with some expert pizza maker, so that you are confident of your skills in preparing the dough and cooking it. You come up with a dish that is satisfying to you, hence in front of it you utter the crucial performative: "This is pizza!" By itself, however, the performative does not suffice to make that dish a pizza. An appropriate expertise is also needed - and you have that. Yet, there must be room also for the possibility of error. Maybe this time you failed and you made a dish that comes close to being pizza, but not enough to count as an instance of the recipe. Some criteria should be spelled out for determining whether a dish authentically renders a recipe. I distinguish two dimensions along which the authenticity of a dish rests: fit and the approval rating of a dish. I examine them in order.

difference between the cooking classes offered by Julia Child on WGBH and the sportive element underscoring most contemporary cooking shows (cfr. also Pollan 2009). 
The fit of a dish is the ratio of two factors: resemblance and context. It is obviously important the extent within which a dish resembles the recipe it purports to render and the other dishes instantiating the recipe. More difficult, however, is to devise the different patterns of resemblance that are relevant for the authenticity of a dish. If instances of a recipe may differ significantly (e.g. wood oven pizza or electric oven pizza; corn flour pizza or wheat flour pizza), it matters which instance the cook takes as a model to imitate; along the same lines, it matters what version of the recipe guides the cook in delivering the dish (e.g. a recipe specifying types of water or the temperature of the plates where to serve the pizza). Yet again, we find here an analogy with music (cfr. Varzi 2013), where - for instance - a song may be the cover of the very first recording of a song or, rather, the cover of a cover of the first recording. Typically, it is up to the cook to specify which dishes and versions of a recipe she intends to imitate. But, as it is often the case also with music, cooks may fail to reveal their specific sources of inspiration, intentionally or more simply because they are not aware of them or have forgotten them. Imitation is most common in art; so is in the kitchen. Through the thousands of recipe books available today, we may attempt to reconstruct key patterns of imitation followed by cooks.

Whether a dish fits as an authentic rendition of a recipe depends not just from how much it resembles the recipe and other dishes that instantiate it; the degree of resemblance should be evaluated vis-à-vis the conditions under which the dish is prepared, such as the expertise of the cook, the types of table companions, and the availability of ingredients and utensils. Thus, for instance, if a cook is reknown for her renditions of a given recipe, our expectations may be higher. Or, if the table companions cannot tolerate high levels of spiciness for red pepper, the cook may refrain from using it in quantities that would be suggested by the recipe and by the dishes that she intends to imitate. Or, consider preparing a recipe after some drastic change in the culinary niche, as after an environmental catastrophe or a diaspora. The distress of the sudden change is augmented by the struggle to preserve a cultural heritage, of which food is a major component. When a people leaves a land for another, for example, they wish to bring their culture along, including their foods. In those circumstances, whether a dish prepared in the new culinary niche authentically reproduces a recipe should be evaluated vis-à-vis the exceptional conditions (cfr. Borghini 2014b).

As for the approval rating, it is typically up to a collectivity of people to assess whether a dish authencally produces a recipe. The higher the approval that a certain dish authentically renders a recipe, the more the identity of the dish will be secured. Subtle methods of measurement for approval can be introduced. As for wines and restaurants, opinions can be weighed based on the degree of expertise of the evaluator. What counts as expertise in this context may be a matter of controversy. Although food critics review dishes they have never prepared before, food critics are supposed to be well-informed about the history of the recipes they judge. On the other hand, it is debatable whether say - a person grown up in the culinary culture of New England, who tasted jambalaya occasionally, can have a saying on the authenticity of a purported rendition of the recipe. 
Fit and approval rating, I argue, provide a sufficiently robust theoretical frame to reconstruct the history of a recipe. Recipes embed a constant struggle between past and future, tradition and innovation. Preparing a recipe embeds much more meaning than "fueling" a stomach; it is an exercise of historical creativity, with an eye to the table companions and another to how the cook wishes to be positioned in society. As cooks aim at imitating previous dishes, a recipe co-evolves with the dishes instantiating it. The recipe and the dishes are intertwined, and a recipe can influence the dishes instantiating it as much as the latter can influence the first. With time, a recipe is typically bound to branch out into different versions. The diners can at this point utilize the recipe to create social boundaries, declaring the different versions as separate recipes. In the case of the chickpea cake quoted above, for instance, we can devise an ecumenic faction, who claims that all versions of the cake (cecina, torta, socca, farinata, fainè, faina, ...) comprise a unique recipe; against it, many local factions affirming the difference of its own recipe. The identity of a recipe can become, hence, a reason of social tension. Without a theory of recipes, however, we have little ground to substantiate disputes regarding them. It is in this sense remarkable how "food wars" and the issues related to food sovereignty (cfr. Dieterle 2015 and Kish 2011) have so far been engaged with a thin theoretical understanding of their key entities - recipes.

The relevance of clarifying our disputes circa the identity of a recipe is best exemplified by geographical indications. In this case, renditions of a recipe are deemed as non-authentic when the ingredients do not originate within a certain region. Thus, the grapes for Champagne or Chianti wines must come from specific lands, or the milk for Parmigiano or Manchego must be produced by livestock (respectively cows and sheeps) inhabiting specific lands. On the other hand, Parmigiano- and Manchego-style cheeses are produced worldwide. By law, those cheeses instantiate a different recipe than Parmigiano and Manchego, and indeed cannot bear those names. But, some are pressing for recognizing selected Parmigiano- and Manchego-style cheeses produced outside of the region of denomination as authentic renditions of the Parmigiano and Manchego recipes. Conversely, the European Union has recently asked to "ban American cheese makers from using terms like parmesan, asiago, feta, gruyere, gorgonzola, fontina, romano and others that refer to European regions from which those cheeses originate," as reported in the Times by Josh Sanburn on March 12, 2014. ${ }^{11}$ The new had many on the Western side of the Atlantic infuriated; but, unfortunately, they had no well-formed theory of the identity of recipes to back up their claims.

The authenticity of recipes is in the majority of cases not a matter of their origin. Recipes evolve. Their authenticity reflects the shift in fitness of the dishes instantiating them as well as the varying conditions for their approval rate. Actually, of most recipes we do not have an author and we have only legendary origins. For instance, for most of the cheeses cited above whose identity is under dispute we have little clues as to the origin and the earliest part of their history. Furthermore, even when the recipe bears the

${ }^{11}$ The full article can be retrieved at $<$ http://time.com/22011/europes-war-on-americancheese/>. 
name of the author, the recipe's identity is not necessarily fixed by that act of original creation. For instance, although fettuccine Alfredo are named after Italian chef Alfredo di Lelio, the standard of authentic fettuccine Alfredo differs quite considerably from the dish prepared by di Lelio at his restaurant Alfredo in Rome during the 1910s and 1920s; and even the "Roman lineage" of Alfredo's recipe is today contended by two restaurants, each with its own rendition, Alfredo alla scrofa and Il vero Alfredo. So is that some regard an interpretation of a recipe as legitimate, while others will consider it disruptive.

\section{§5.3 Open-Endedness}

I must finally consider an aspect of recipes that does not call for as much discussion as expertise and authenticity, but that nonetheless is of high importance. According to the account I offered, recipes are tied to history because they depend upon the dishes that instantiate them for their existence (the Aristotelian view defended in \$4) and for their identity (the view of authenticity put forward in §5.2). As instances of a recipe keep being delivered, the recipe is bound to change. The evolution of a recipe rests on a complex historical process driven by multiple variables, including the creativity of the cooks, the opinions of the diners, and the conditions under which the recipe will be prepared. For each recipe, the possible trajectories of evolution are countless. Additionally, no requirement of spatio-temporal continuity seems to be in place too, so that - e.g. following the discovery of an inscription - a cook may revive a long-gone recipe.

All those clues point to the conclusion that recipes are open-ended entities. This is not to suggest that we cannot identify a recipe because its existence is not yet over. We can identify the instances of pizza, of fettuccine Alfredo, and of the uncounted other recipes that humans have come up with. But, we are looking at a part - not at the whole of those recipes. Thus, we cannot identify all the instances of those recipes, if by "all" we mean also future instances; because we do not know yet how the recipes will evolve and because their possible instances are countless. Analogously, we can identify an instance of the song A Groovy Kind of Love; but we have yet to experience its future instances, and those we cannot identify because there are countless possible ways in which the song may evolve. In other words, I am defending an anti-essentialist view of recipes, in that I am arguing that the identity of a recipe cannot be tied to any property that some of its instances possesses at any moment of its history.

James Carse's study of games suggests a succinct rendition of the open-ended character of recipes (Carse 1986). Carse distinguishes between finite and infinite games. In finite games, the rules specify also the conditions for the end of the game; this is the case, for instance, in a boxing match, or a football game, or in the pursuit of a Ph.D. degree in philosophy. In infinite games, instead, the rules specify no initial or ending conditions; the game is played for the sole purpose of playing it. Each recipe is an infinite game, whose rules - i.e. expertise, performative utterance, collective judgment - are known, but whose beginning and end may remain unknown. Cooking, more generally, is the game of producing recipes, and this paper is an attempt to clarify the rules of the game. 


\section{§6 Conclusions}

In this paper I aimed at laying out the main infrastructure of a theory of recipes. Along the way, I proposed a constructivist account of recipes, which rivals the realist accounts implicitly suggested by current policies. As recipes acquire an increasingly important socio-economic role, it is pivotal that the main issues here tackled be studied in greater details. The account I endorse arises out of years spent alongside food producers, cooks, food activists, policy makers, and food educators. The account, I contend, helps accommodating the tensions that arise out of current debates circa the identity of recipes and can be used to orient policy-makers, lawyers, scholars and educators in approaching issues surrounding food and identity. I cursorily pointed out why this is the case, but further work is required to study the links between theory, policy, and practice in this field. $^{12}$

\footnotetext{
${ }^{12}$ I thank an anonymous referee, Naomi Arbit and Carolyn Richardson for helpful comments on a previous version of this paper. Research for the paper grew out of a long period of confrontation with colleagues and friends, including: Andrea Baldini, Dario Cecchini, Andrea Falaschi, Christia Mercer, Nicola Perullo, Bridget Potter, Gus Rancatore, Achille Varzi, Merry "Corky" White, and Ben Wurgaft. I wish to thank also the students in the seminars on the philosophy of food that I taught over the past seven years at the College of the Holy Cross and at the University of Gastronomic Sciences.
} 


\section{References}

Allhoff, Fritz \& Marcus P. Adams (2010) (eds.), Whiskey and Philosophy: A Small Batch of Spirited Ideas, Oboken, NJ: Wiley

Boorstin, D. J. (1964), The Image. A guide to Pseudo-Events in America, New York: Harper

Borghini, Andrea (2014a), 'Geographical Indications, Food, and Culture,' in P.B. Thomson and D.M. Kaplan (eds.), Encyclopedia of Food and Agricultural Ethics, Springer: $1115-1120$

Borghini, Andrea (2014b), 'Authenticity in Food,' in P.B. Thomson and D.M. Kaplan (eds.), Encyclopedia of Food and Agricultural Ethics, Springer: 180-185

Borghini, Andrea (2014c), 'Substantial Equivalence,' in P.B. Thomson and D.M. Kaplan (eds.), Encyclopedia of Food and Agricultural Ethics, Springer: 1669-1673

Borghini, Andrea (2012), 'On Being the Same Wine' Rivista di estetica 51: 175-192

Borghini, Andrea (2011), 'What Is a True Ribollita? Memory and the Quest for Authentic Food,' in T. Piazza (ed.), Secret and Memory in the Information Age, Afrontamento, Porto, 2011: 93-106

Burnham, Douglas \& Ole M. Skilleas (2012), The Aesthetics of Wine, Oxford: WileyBlackwell

Carse James P. (1986), Finite and Infinite Games. A Vision of Life as Play and Possibility, Ballantine Books, New York.

Casati, Roberto \& Achille C. Varzi (2007), "Foreword," The Monist 90: 331-2

Cohen, E. (2002), "Authenticity, Equity and Sustainability in Tourism," Journal of Sustainable Tourism 10: 267-276

Collins, Harry (2013), "Three Dimensions of Expertise," Phenomenology and Cognitive Sciences 12: 253-273

Coombe, Rosemary (2009), "The Expanding Purview of Cultural Properties and Their Politics," Annual Review of Law and Social Science 5: 393-412

Davies, David (2004), Art as Performance, Malden MA: Blackwell

Dieterle, Jill M. (2015) (ed.), Just Food. Philosophy, Justice, and Food, London: Rowman \& Littlefield

Dodd, Julian (2007), Works of Music. An Essay in Ontology, Oxford: Oxford University Press

Doolittle, Ford W. \& Thane R. Papke (2006), "Genomics and the Bacterial Species Problem," Genome Biology 7: 116

Ereshefsky, Mark (2010), "Microbiology and the Species Problem," Biology and Philosophy, 25: 553-568

Fischler, Claude (1988), "Food, Self, and Identity," Social Science Information 27: 275292

Fischler, Claude (1980), "Food Habits, Social Change, and the Nature/Culture Dilemma," Social Science Information 19: 937-953

Franklin, Laura R. (2007), "Bacteria, Sex, and Systematics," Philosophy of Science 74: 69-95

Goodman, Nelson H. (1976), The Languages of Art. An Approach to a Theory of Symbols, 2nd edition, Indianapolis: Hackett Publishing Company 
Gatti, Antonietta and Stefano Montanari, 2007, Nanopathologies, Singapore: Pan Stanford

Gaut, Berys (2000), "'Art' as a Cluster Concept" in N. Carroll (ed.), Theories of Art Today, Madison: University of Wisconsin Press: 25-44

Germann Molz, Jennie (2004), "Tasting an Imaginary Thailand: Authenticity and Culinary Tourism in Thai Restaurants," in L. Long (ed.), Culinary Tourism, Lexington: The University Press of Kentucky: 53-75

Hales, Steven D \& Michael C. Jackson (2008) (eds.), Beer \& Philosophy. The Unexamined Beer Isn't Worth Drinking, Malden: Blackwell Publishers

Jackson, Peter (1999), "Commodity Cultures: The Traffic in Things," Transactions of the Institute of British Geographers 24: 95-108

Kish Zenia (2011), "Food Sovereignty," in D.K. Chatterjee (ed.), Encyclopedia of Global Justice, New York: Springer: 352-358

Kivy, Peter (1995), Authenticities: Philosophical Reflections on Musical Performance, Ithaca: Cornell University Press

Kivy, Peter (1993), The Fine Art of Repetition: Essays in the Philosophy of Music, Cambridge: Cambridge University Press

Korsemeyer, C. (1999), Making Sense of Taste: Food and Philosophy, Ithaca, NY: Cornell University Press

Limberg H. (2009), Ruth's Rhyming Recipes for Family Fun, Bloomington, IN: AuthorHouse

Perullo, Nicola (2012), "Wineworld: Tasting, Making, Drinking, Being," Rivista di estetica $51: 3-48$

Pollan, Michael (2009), "Out of the Kitchen, Onto the Couch," The New York Times, August 2

Scruton, Roger (2009), I Drink Therefore I Am: A Philosopher's Guide to Wine, New York: Continuum

Shapiro, Laura (2008), Perfection Salad. Women and Cooking at the Turn of the Century, Berkeley, CA: University of California Press

Sims, Rebecca (2009), "Food, Place and Authenticity: Local Food and the Sustainable Tourism Experience" Journal of Sustainable Tourism 17: 321-336

Smith, Barry C. (2007) (ed.), Questions of Taste: The Philosophy of Wine, Oxford: Oxford University Press

Telfer E. (1996), Food for Thought: Philosophy and Food, New York: Routledge

Todd, Cain (2010), The Philosophy of Wine: A Case of Truth, Beauty, and Intoxication, Durham: Acumen

Varzi, Achille C. (2013), "Cover to Cover," Current Musicology 95: 177-191

Yeung, May T. (2014), "Geographic Indications," in Paul B. Thomson and David M. Kaplan (eds.), Encyclopedia of Food and Agricultural Ethics, Springer, 2014: $1107-$ 1115

Wang, Ning (1999), "Rethinking authenticity in the tourist experience," Annals of Tourism Research 26: 349-370 